Archives

$00 \mid 1988$

Journée d'études Werner Sombart

\title{
Werner Sombart et Max Weber
}

\section{Freddy Raphael}

\section{(apenEdition \\ Journals}

\section{Édition électronique}

URL : http://journals.openedition.org/ccrh/2981

DOI : $10.4000 /$ ccrh.2981

ISSN : $1760-7906$

\section{Éditeur}

Centre de recherches historiques - EHESS

Édition imprimée

Date de publication : 14 mars 1988

ISSN : 0990-9141

\section{Référence électronique}

Freddy Raphael, «Werner Sombart et Max Weber », Les Cahiers du Centre de Recherches Historiques [En ligne], 00 | 1988, mis en ligne le 13 avril 2009, consulté le 19 avril 2019. URL : http:// journals.openedition.org/ccrh/2981; DOI : 10.4000/ccrh.2981

Ce document a été généré automatiquement le 19 avril 2019

(ㄷ) Tous droits réservés 


\title{
Werner Sombart et Max Weber
}

\author{
Freddy Raphael
}

\section{Le socialisme de la chaire}

1 On a pu définir les relations entre Max Weber et Werner Sombart comme celles de deux frères ennemis ${ }^{1}$. Ces deux universitaires allemands, qui avaient le même âge, à un an près, menèrent des recherches parallèles sur la formation de l'esprit du capitalisme moderne. Leurs travaux dans ce domaine s'entrecroisèrent, mais chacun d'entre eux, tout en critiquant les travaux de l'autre, reconnaissait ce qu'il lui devait. Dans L'éthique protestante et l'esprit du capitalisme, Max Weber parle en termes élogieux des "exposés souvent heureux et efficaces de Sombart ", surtout quand il se réfère à Der moderne Kapitalismus dans la première édition de 1902. Il souligne tout ce que ses analyses doivent « aux grands ouvrages de Sombart et à leurs formules pénétrantes -et cela même lorsqu'elles empruntent une voie différente»(p.61), et remarque que «Sombart n'a nullement négligé l'aspect éthique de l'entrepreneur capitaliste» (p. 51). Il ajoute ${ }^{2}$ que ceux qui se sentent en désaccord permanent et déterminé avec les vues de Sombart, et rejettent certaines de ses thèses, n'en doivent pas moins rester convaincus de l'importance de son oeuvre. Mais, d'autre part, Weber n'est pas tendre à l'égard de l'ouvrage de Sombart, Le bourgeois, dans les longues notes « anticritiques » qu'il lui consacre dans l'édition de 1920 de L'éthique protestante. Quant à Sombart, il indique dans Les juifs et la vie économique que ce sont les études de Max Weber qui l'ont incité à entreprendre ses recherches sur le judaïsme. Mais, ajoute-t-il aussitôt, si le puritanisme a pu avoir une influence positive sur le développement du capitalisme, c'est seulement dans la mesure où il a repris des idées qui se trouvent exprimées avec beaucoup plus de force et de relief dans la religion juive ${ }^{3}$. "Après avoir pris connaissance de ses recherches, je me suis demandé (et je crois que tout observateur attentif était en droit de se poser la même question) si ce que Weber attribue au puritanisme n'a pas été accompli, après et avant lui, par le judaïsme ; bien plus : si même ce que nous appelons puritanisme n'est pas, dans ses traits essentiels, de nature judaïque $»^{4}$. 
2 En 1904, Max Weber et Werner Sombart fondèrent, avec Edgar Jaffé, l'Archiv für Sozialwissenschaft und Sozialpolitik, qui s'inscrivait dans le prolongement d'une ancienne revue, l'Archiv für soziale Gesetzgebung und Statistik, dont Heinrich Braun avait été le fondateur en 1888. Le comité de rédaction de l'Archiv für Sozialwissenschaft und Sozialpolitik comprenait au départ Max Weber, Edgar Jaffé et Werner Sombart. D'une lettre de Max Weber du 17 juillet 1903, citée par Marianne $W^{2} e^{5}$, il ressort qu'Edgar Jaffé fut le véritable promoteur de la revue. Il venait d'achever ses études par une "Dissertation » portant sur « Die Arbeitsteilung im englischen Bankwesen » (1902). Plus tard, il deviendra le ministre des finances du gouvernement bavarois de 1918 présidé par Kurt Eisner. Heinrich Braun dirigea également, de 1892 à 1895, le Sozialpolitisches Zentralblatt, et de 1911 à 1913 les Annalen für Sozialpolitik und Gesetzgebung. Au moment d'entrer dans le comité de rédaction de l'Archiv, W. Sombart avait publié Der Sozialismus und die soziale Bewegung (1897) et Der moderne Kapitalismus (1902). Pour Max Weber, l'Archiv für Sozialwissenschaft und Sozialpolitik fut, pendant un certain temps, le principal instrument de communication et d'échange avec le monde intellectuel, alors que la maladie nerveuse l'empêchait d'enseigner. De 1906 à 1910, Werner Sombart vint souvent rendre visite à Max Weber dans sa maison de Heidelberg. De même, il fut l'un des rares à soutenir certaines positions de Weber devant l'Association allemande de sociologie. M. Weber sut reconnaître les mérites de $\mathrm{W}$. Sombart, tout en étant parfaitement conscient des profondes divergences de vues qui l'opposaient à ce dernier, et il collabora avec lui de nombreuses années.

Au lendemain de la fondation de l'Empire, la pensée allemande était plus attirée par l'idée d'un dépassement lointain de l'univers industriel que par la recherche de solutions aux problèmes immédiats. Néanmoins, il y eut un certain nombre d'intellectuels, et en particulier de professeurs d'économie, qui se préoccupèrent de la condition ouvrière. Adversaires du socialisme, ils recherchèrent les moyens d'améliorer cette condition à l'intérieur du régime capitaliste dont ils demandaient à l'État de corriger les abus. Ils se regroupèrent en 1872 au sein du Verein für Sozialpolitik (Association pour une politique sociale), qui s'efforça de promouvoir une réforme dans le sens du progrès social, mais condamnait le recours à la violence et à la révolution. Le " Socialisme de la chaire ", ainsi que l'appelèrent par dérision ses adversaires, s'en prenait à l'École de Manchester et à ses adeptes allemands, c'est-à-dire essentiellement au parti national-libéral. C'est moins l'éphémère Association pour une politique sociale (Verein für Sozialpolitik), née en 1872, qui exerça une influence en Allemagne, que les écrits, l'enseignement et les polémiques de quelques professeurs. "Politiquement, certains étaient des conservateurs, comme Adolf Wagner, d'autres, des progressistes, comme Gustav Schmoller, ou des catholiques, comme Lujo Brentano. Ils défendaient en commun l'idée que l'État avait une responsabilité à l'égard des ouvriers et ne pouvait laisser jouer les seules lois de l'économie de marché quand il s'agissait du sort des hommes. Sur le rôle qui incombait à l'État, ils étaient loin d'être unis; les uns attendaient surtout une législation sur les salaires et les conditions de travail, d'autres un arbitrage dans les conflits du travail, d'autres encore une action en faveur des coopératives ouvrières. Ils n'eurent guère d'influence sur les milieux ouvriers, mais ils fournirent peut-être à Bismarck certaines des idées qui inspirèrent sa politique sociale, et en tout cas le vocabulaire avec lequel il les présenta au Reichstag $»^{6}$. 


\section{Éléments de la méthodologie de Max Weber et de Werner Sombart}

\section{Sociologie et histoire}

4 L'intelligibilité historique a pour vocation, selon W. Dilthey, d'analyser des actes ou des institutions guidés « par des intentions $»^{7}$ et, de ce fait, liés à des valeurs. Dans Le monde de $l^{\prime} e s p r i t^{8}$, il souligne que ce qui est se révèle inséparable du sentiment de sa valeur et de son idéal: «Ce qu'il y a d'essentiel dans les manifestations de la vie est l'expression du système vivant des valeurs qui est en elles, et cet essentiel s'exprime à son tour par des idéaux et des normes qui règlent de l'intérieur les manifestations de cette vie ». Max Weber refuse au jugement de valeur le droit d'existence au sein de la science, mais il n'exclut nullement le rapport aux valeurs. Si Max Weber s'est interrogé dès 1904 sur le statut scientifique des sciences sociales ${ }^{9}$, ce n'est que dans ses écrits tardifs que le terme de Wertfreiheit, que Julien Freund ${ }^{10}$ a traduit par "neutralité axiologique", devient un concept majeur. La neutralité axiologique a pour rôle de nous mettre en garde contre l'impérialisme d'une valeur et de l'activité qu'elle supporte, « non seulement parce qu'il n'y a pas de doctrine ou de conception du monde définitive, mais aussi parce que chaque valeur prend historiquement des significations variables suivant les époques». L'économisme en arrive à ignorer, à nier et à dénaturer tout phénomène qui résiste à son interprétation univoque. En fait, pour Max Weber, on ne peut même pas expliquer par la seule économie tous les phénomènes économiques. "Quel que soit le domaine des manifestations culturelles, la réduction aux seules causes économiques n'est exhaustive en aucun sens, pas même dans celui des phénomènes proprement économiques ${ }^{11}$. Le rapport aux valeurs commande l'interprétation des événements et des phénomènes, y compris l'imputation causale et la construction des idéaux-types. « Au fond, c'est parce que le rapport aux valeurs est sans cesse présent dans le travail du spécialiste qu'aucune explication ne sera jamais définitive, et qu'elle sera toujours et nécessairement interprétative. Les sciences économiques et sociales sont inévitablement des herméneutiques. D'où l'importance que Weber attache au Verstehen : il arrive toujours un moment où l'explication purement causale est bloquée et où il n'y a plus d'autre moyen de s'en sortir qu'en y associant la compréhension interprétative $»^{12}$. Il convient donc de reconnaître que, dans les sciences sociales, les explications fournies ne le sont jamais qu'à partir de points de vue qui sont incapables de restituer intégralement le cours réel des choses. Le rapport aux valeurs est une manière d'évaluer théoriquement les événements et les phénomènes. D'où la nécessité de respecter la neutralité axiologique pour ne pas faire passer cette évaluation théorique pour une prise de position personnelle ou partisane. «Elle indique au chercheur qu'il est lui-même prisonnier de certaines valeurs, qu'il doit en prendre conscience pour ne pas les substituer implicitement aux valeurs que représentent les hommes et les événements qu'il étudie. Autrement dit, il doit éviter d'élever au rang d'une connaissance scientifique ce qui n'est que l'expression de ses convictions personnelles $»^{13}$. Il n'existe pas de rapport aux valeurs universelles, d'où l'on pourrait déduire la réalité ou encore auquel on pourrait la réduire. Il n'y a pas d'objectivité absolue, puisque l'objectivité est elle-même fondée sur des valeurs. «Si la neutralité axiologique met le savant en garde contre la tentation de faire passer ses convictions personnelles pour des résultats de la recherche, elle exige également qu'on ne fasse pas passer la valeur pour de l'être, par confusion de la ratio cognoscendi avec la 
ratio essendi. Cela veut dire qu'elle refuse toute substantification de la valeur par l'universalisation d'un rapport aux valeurs déterminé et particulier. Toute valeur est de jugement $»^{14}$.

W. Sombart établit, comme M. Weber, une séparation radicale entre les jugements de valeur et l'explication scientifique. Lors de l'assemblée de l'Association pour la politique sociale à Vienne en 1909, il déclare: «Nous ne pouvons pas discuter de ces sortes de jugements tant que l'on n'a pas prouvé scientifiquement qui des blondes et des brunes sont les plus charmantes». Max Weber lui fait écho dans son étude sur la "neutralité axiologique » lorsqu'il écrit : " A dire vrai, il n'est nullement question de discuter ici dans quelle mesure des évaluations pratiques, en particulier celles d'ordre éthique, peuvent prétendre à une dignité normative, c'est-à-dire prendre un autre caractère que la question posée par l'exemple suivant : faut-il préférer les brunes ou les blondes? ou par d'autres jugements subjectifs analogues au goût. Ces problèmes appartiennent à la philosophie des valeurs, non à la méthodologie des disciplines empiriques $»^{15}$. W. Sombart prétend, lui aussi, faire preuve d'objectivité et présenter des essais qui répondent «à la réalité des faits ${ }^{16}$. Il affirme que son ouvrage sur Les juifs et la vie économique n'est pas "un livre à thèse ", car il n'entend pas faire prévaloir une certaine conception de l'histoire ; c'est un « livre rigoureusement scientifique», qui «se borne à constater et à expliquer les faits et s'abstient de tout jugement de valeur $»^{17}$.

6 Pour appliquer le principe de la neutralité axiologique, Max Weber a recours à l'idéaltype. Il ne s'agit nullement d'un concept qui permettrait de juger de la valeur d'un phénomène, ou encore de l'hypostasier. C'est un instrument destiné à mieux le faire connaitre, sans chercher à en donner une description intégrale. L'idéaltype n'est qu'un moyen, toujours révisable et susceptible de correction, destiné à mettre de l'ordre dans le chaos des faits et à les rendre intelligibles ${ }^{18}$. La conception partielle et analytique de la causalité nécessite, elle aussi, la sélection de certains aspects du phénomène, que l'on juge significatifs pour la recherche, afin de cerner, par l'élaboration de différents modèles, une réalité complexe et confuse. Il s'agit d'une construction cohérente et rigoureuse, mais qui ne saurait maitriser la diversité infinie du réel. Dans tous les cas, c'est le rapport aux valeurs choisi qui commandera la sélection des faits, sans que l'un de ces points de vue soit exclusif des autres. La faute épistémologique consisterait à attribuer, en dernière analyse, une validité supérieure à l'un des points de vue sur les autres.

C'est à son ami, le juriste Georg Jellinek, que Weber emprunte le concept de l'idéaltype, que Georg Simmel développera également. Mais auparavant déjà, l'École néo-kantienne de Bade, représentée par Windelband, Rickert et Lask, considérait la science historique, qui s'intéresse autant au singulier qu'au général, non pas comme une reproduction ou une copie du réel, mais comme une reconstruction conceptuelle. Heinrich Rickert ${ }^{19}$, affirmait que le réel est inépuisable, parce qu'il est doublement infini, intensivement et extensivement, alors que la connaissance est toujours bornée par les conditions mêmes de son exercice, à savoir son appareil de concepts. «La science... ne peut embrasser la totalité du réel; elle ne peut donc en être la copie (Abbildung), mais elle est la transformation (Umbildung) du réel, dans le moment même où elle forme des concepts ( Begriffsbildung) pour l'appréhender ». Dans son ouvrage Allgemeine Staatslehre, qui date de 1900, Georg Jellinek oppose au type-empirique le type idéal, "instrument heuristique » qui ne se limite pas à reproduire servilement la réalité, mais qui élabore, subjectivement certes, des modèles. Dans sa Philosophie des Geldes, que Max Weber a lue peu de temps avant d'écrire son étude sur L'objectivité de la connaissance dans les sciences et la politique 
sociales, G. Simmel ${ }^{20}$ rappelle que la plupart du temps le sociologue élabore des concepts, dont le caractère "pur et absolu» n'a pas son répondant sur le plan de l'expérience. "Cette méthode particulière, qui consiste à construire des concepts en sélectionnant les traits et en les grossissant, livre une connaissance du monde qui correspond à notre faculté de compréhension... Notre intellect n'est capable de saisir la réalité qu'à l'intérieur des limites de purs concepts, qui, peu importe s'ils s'éloignent de la réalité, attestent de leur légitimité dans la mesure où ils nous permettent d'interpréter cette réalité ».

8 Tout examen attentif portant sur les éléments conceptuels d'un exposé historique montre que l'historien, dès qu'il cherche à s'élever au-dessus de la simple constatation de relations concrètes pour déterminer la signification culturelle d'un événement singulier, si simple soit-il, donc pour le caractériser, «travaille et doit travailler avec des concepts qui, en général, ne se laissent préciser de façon rigoureuse et univoque que sous la forme d'idéaltypes ». A la description sans présupposition d'un phénomène concret «isolé », dont la singularité ne permet aucune étude comparative, Max Weber oppose « la synthèse abstractive" (Abstrahierende Zusammenfassung) de ce qui est commun à plusieurs phénomènes concrets. Il est impossible de saisir avec suffisamment de précision les aspects singuliers d'un phénomène au moyen de concepts génériques, parce que, par nature, ils n'appréhendent que le général. L'idéaltype est une construction intellectuelle qui a pour rôle de remédier à cette carence; il est élaboré pour saisir la singularité historique et sociologique d'un phénomène. Julien Freund ${ }^{21}$ définit l'idéaltype comme un tableau de pensée, à la fois rationnel et utopique, qui regroupe de façon cohérente divers éléments d'une réalité empirique, donnés d'une manière diffuse, discrète et isolée, pour les mettre en évidence sous un point de vue déterminé. Pour définir des concepts univoques, la sociologie est contrainte d'élaborer des types "idéaux " purs à partir des différentes structures historiques, économiques et religieuses, "qui révèlent alors chacune pour soi l'unité cohérente d'une adéquation significative aussi complète que possible, mais qui, pour cette raison, ne se présentent peut-être pas plus dans la réalité sous cette forme pure, absolument idéale, qu'une réaction physique que l'on considère sous l'hypothèse d'un espace absolument vide $»^{22}$.

Werner Sombart prône également le recours à l'idéaltype. Mais dans Die drei Nationalökonomien $^{23}$, il reproche à Max Weber, qui a, selon lui, élaboré ce concept, de ne pas l'avoir défini avec précision, dans une perspective logique libre de tout préjugé. La meilleure preuve en est qu'à la suite de Max Weber, on a appliqué indistinctement le concept d'idéaltype à des phénomènes sociaux ou religieux, tels le christianisme ou le capitalisme moderne, et à des personnages de l'histoire. A ces derniers W. Sombart dénie toute possibilité de constituer des types. Il oppose, quant à lui, le «type idéal » au «type réel ». Le premier exprime, dans toute sa pureté, «l'essence » (Wesen) de l'objet étudié ; pour cela, il convient d'écarter les éléments accessoires et d'accentuer (Steigerung) les traits significatifs essentiels (Wesensmerkmale). Dans l'élaboration d'un idéaltype tout jugement de valeur doit être banni. Par contre, le type réel saisit l'aspect contingent et historique de l'objet étudié ; on le construit à l'aide d'éléments empiriques, de traits accidentels et relatifs. 


\section{Max Weber et Werner Sombart confrontés au matérialisme historique}

On s'est basé sur une lecture hâtive de l'oeuvre de Max Weber, et sur la déclaration imprudente qu'il fit aux étudiants de l'université de Vienne en 1918, lorsqu'il présenta son cours comme une "réfutation positive du matérialisme historique ", pour réduire l'analyse wébérienne à une réfutation du marxisme, à une tentative pour faire dériver les comportements économiques de la conscience religieuse. Certes, dans L'éthique protestante et l'esprit du capitalisme, il dénonce " la doctrine simpliste du matérialisme historique ${ }^{24}$ et les idées superficielles des «théoriciens de la superstructure ${ }^{25}$. Mais s'il se moque de «ceux dont la bonne conscience causale ne peut se passer d'interprétation économique ou matérialiste $\aleph^{26}$, il précise cependant qu'il tient " pour fort importante l'influence du développement économique sur le destin des idées religieuses ». Max Weber fut l'un des rares universitaires de son époque à avoir insisté sur l'apport de Marx, et notamment sur son analyse du caractère déterminant, suivant les conditions, de l'économie et de la lutte de classes. Néanmoins, il a toujours refusé ce qu'il appelle la métaphysique de Marx, à savoir que les phénomènes de production seraient seuls déterminants en dernière analyse. "Il reconnaît une valeur idéaltypique, c'est-à-dire hypothélique aux idées de Marx, mais il conteste le fait qu'elles puissent figurer la réalité. En effet, en raison de l'infinité intensive et extensive du réel, celui-ci reste toujours au-delà de toute construction conceptuelle, qui est nécessairement finie et limités à cause des présuppositions de départ. Aussi range-t-il certaines explications de Marx dans la catégorie des hypostases $»^{27}$. Weber dénonce le monisme causal du marxisme. Celui-ci refuse de faire de l'histoire « un sujet métaphysique, auquel des individus humains réels serviraient de simples supports ", car c'est l'homme qui, par la praxis, engendre sa propre histoire. Cependant, toutes les activités humaines sont conditionnées matériellement: "Même les fantasmagories dans le cerveau humain sont des sublimations résultant nécessairement $\mathrm{du}$ processus de leur vie matérielle, que l'on peut constater empiriquement et qui repose sur des bases matérielles. De ce fait, la morale, la religion, la métaphysique et tout le reste de l'idéologie, ainsi que les formes de conscience qui leur correspondent, perdent aussitôt toute apparence d'autonomie. Elles n'ont pas d'histoire, elles n'ont pas de développement; ce sont au contraire les hommes qui, en développant leur production matérielle et leurs rapports matériels, transforment, avec cette réalité qui leur est propre, à la fois leur pensée et les produits de leur pensée. Ce n'est pas la conscience qui détermine la vie, mais la vie qui détermine la conscience $»^{28}$. Karl Marx a longuement traité dans ses premières oeuvres des rapports entre la religion et l'économie, mais d'une façon idéologique et polémique. «En faisant de la religion une superstructure en réaction dialectique avec le substrat économique, il a faussé le problème par une réduction que même la métaphysique ne saurait justifier. Il a ainsi manqué le problème de l'essence du phénomène religieux en le privant de toute autonomie dans l'activité. De plus, le rapport qu'il a établi entre les deux ordres est tellement général qu'il ne peut donner lieu qu'à un dogmatisme de philosophe et non à des recherches historiques ou sociologiques concrètes. Enfin, en se posant comme adversaire de la religion et du capitalisme, il s'est mis dans une mauvaise situation pour comprendre les relations positives qui ont pu exister historiquement, dans toutes leurs variations, entre ces deux activités $\aleph^{29}$. Au premier congrès de l'Association des sociologues allemands, en 1910, M. Weber s'écria: «J'entends protester contre la 
déclaration de l'un des orateurs, selon laquelle un seul facteur, que ce soit la technologie ou l'économie, peut constituer la cause "ultime» ou »réelle» d'un phénomène. Si nous considérons les différentes chaînes causales, nous remarquons que parfois l'on passe des facteurs politiques à des facteurs politiques et économiques, et parfois des facteurs politiques à des facteurs religieux et économiques, etc... Tout ceci évolue sans cesse. J'estime que l'affirmation du matérialisme historique, selon laquelle l'économique est, dans un certain sens, le point ultime dans la filiation causale, n'est plus du tout recevable dans une perspective scientifique $\aleph^{30}$. Pour Weber, n'importe quelle activité peut, suivant les circonstances, jouer le rôle d'une force de libération ou d'asservissement. Aucune ne possède le privilège ontologique, pas plus l'économie que la politique, d'être exclusivement libératrice. L'erreur de Marx fut d'attribuer le monopole de la libération uniquement à l'économie. Toute activité peut être un frein ou un obstacle au développement, comme elle peut le favoriser. Max Weber affirme qu'à des infrastructures économiques identiques peuvent correspondre des superstructures politiques et idéologiques différentes. Il n'est pas exact, comme le prétend Marx, que le moulin est à la base du féodalisme, et la machine à vapeur à la base du capitalisme : « car l'âge du moulin, qui a duré jusqu'aux temps modernes, a produit toutes sortes de superstructures culturelles dans toutes sortes de domaines ».

11 En somme, ce que Max Weber reproche au marxisme, c'est d'avoir fait de l'économie le fondement du développement historique en lui attribuant le rôle de condition ultime de toute activité humaine. C'est là un jugement de valeur qui n'a rien de scientifique, pas plus que ne le serait la théorie qui ferait de la religion ou de la politique la condition qui déterminerait, en dernière instance, les autres activités humaines. « C'est pourquoi, écritil dans L'objectivité de la connaissance ${ }^{31}$, nous nous bornons à constater que toutes les «lois» et constructions du développement de l'histoire spécifiquement marxistes ont évidemment-dans la mesure où elles sont théoriquement correctes-un caractère idéaltypique. Quiconque a appliqué une fois les concepts marxistes connaît l'importance heuristique éminente, et même unique, de ces idéaltypes quand on les utilise seulement pour leur comparer la réalité, mais aussi leur danger dès qu'on les présente comme des constructions ayant une validité empirique ou comme des forces agissantes réelles.... » Dans la conclusion de L'éthique protestante, il précise que son intention n'est nullement d'opposer à une interprétation causale de l'histoire une interprétation spiritualiste. Les deux interprétations sont possibles et également valables tant qu'elles se contentent de rester des moyens heuristiques, mais elles servent l'une et l'autre aussi mal la vérité historique dès qu'elles prétendent apporter des conclusions définitives. "Cela veut dire que, pour Weber, toutes les conceptions du monde sont valables tant qu'elles se limitent à n'être que des rapports aux valeurs. Elles deviennent dangereuses et antiscientifiques quand elles transforment ce rapport aux valeurs en jugement de valeur, et à ce titre elles contribuent à entretenir l'antagonisme des valeurs. Il ne condamne nullement une vision unilatérale des choses -elle peut être utile et parfois nécessaire -à condition que l'on reste conscient de la relativité du procédé $\aleph^{32}$. Ce que Max Weber reproche à Marx, ce n'est pas d'avoir souligné le rôle que l'économie pouvait jouer en tant que facteur à la fois conditionnant et conditionné, mais de lui avoir attribué le rôle de fonction déterminante en dernière instance. «L'économie est une raison de l'explication des choses, mais non point une raison prépondérante ni suffisante. Ce que Weber récuse, c'est la systématisation d'un rapport de valeur, parce que la systématisation est le résultat d'un jugement de valeur qui privilégie un facteur au nom de la foi ou de l'opinion $»^{33}$. 
12 Julien Freund ${ }^{34}$ a réfuté l'assertion selon laquelle l'oeuvre de Max Weber aurait pour objectif de prendre le contre-pied de l'explication marxiste. Certes, Weber critique le simplisme de certaines formulations de théoriciens du matérialisme historique, mais il vise davantage le marxisme vulgaire de son époque que la philosophie même de Marx. « Il est également vrai qu'il n'accepte pas de faire de la religion une superstructure, en raison de sa conception de la causalité, selon laquelle chaque phénomène est toujours déterminé par des facteurs multiples sans que l'on puisse les subordonner systématiquement à l'un d'entre eux. Sur ce point, il est en désaccord avec l'épistémologie marxiste, parce qu'elle ne répond ni à l'esprit ni aux présupposés de la science. Néanmoins, son essai a non pas une intention négative, à savoir réfuter le marxisme, mais positive, en ce sens qu'il a pour objet à la fois de montrer la complexité de l'explication sociologique et d'éclairer un aspect de la genèse du capitalisme moderne ». En étudiant la conduite significative de l'être religieux, Max Weber ne prétend nullement réfuter le matérialisme historique en faisant dépendre le comportement économique de l'option religieuse, au lieu de voir en celle-ci la superstructure d'une société dont l'infrastructure serait constituée par les rapports de production. «Weber a voulu démontrer que les conduites des hommes dans les diverses sociétés ne sont intelligibles que dans le cadre de la conception générale que ces hommes se sont fait de l'existence; les dogmes religieux et leur interprétation sont parties intégrantes de ces visions du monde $»^{35}$. Il s'oppose cependant à un certain dogmatisme marxiste selon lequel, en dernière analyse, les phénomènes religieux ne sont que le reflet du substrat économique. Weber refuse tout déterminisme unilatéral, car une éthique économique n'est pas une simple fonction de formes d'organisation économique, pas plus qu'inversement elle ne crée celles-ci de manière univoque. Aussi, ne saurait-il être question pour lui de substituer une causalité des forces religieuses à la causalité des forces économiques. «Est-il nécessaire de protester que notre dessein n'est nullement de substituer à une interprétation causale exclusivement matérialiste, une interprétation spiritualiste de la civilisation et de l'histoire qui ne serait pas moins unilatérale ? Toutes deux appartiennent au domaine du possible, il n'en demeure pas moins que, dans la mesure où elles ne se bornent pas au rôle de travail préparatoire, mais prétendent apporter des conclusions, l'une et l'autre servent aussi mal la vérité historique $»^{36}$. L'approche unilatérale du matérialisme historique, qui tend à rechercher les causes économiques des faits historiques, est valable comme méthode d'analyse, mais ne saurait constituer une interprétation globale de l'histoire. Weber insiste sur l'influence réciproque des convictions religieuses et du comportement économique; il montre « comment la conduite religieuse oriente ou conditionne en partie les autres activités humaines et respectivement se trouve conditionnée par elles $»^{37}$. Les rapports entre l'économie et la religion sont trop complexes pour qu'ils se laissent réduire à une causalité unilatérale et mécanique. Pour Weber, l'éthique économique d'un système religieux se définit par des impulsions pratiques pour l'action qui s'enracinent dans les contextures psychologiques et pratiques de la religion. Mais une éthique économique n'est jamais déterminée par la seule religion et elle possède toujours un certain degré d'autonomie véritable. "Des données géographiques, économiques et historiques déterminent cependant, au plus haut degré, l'ampleur de cette autonomie. Quoi qu'il en soit, la détermination religieuse du mode de vie est également un -je dis bien un-des facteurs déterminants de l'éthique économique. Bien entendu le mode de vie religieux est à son tour profondément influencé par les facteurs économiques et politiques, agissant dans des limites géographiques, politiques, sociales et nationales données ${ }^{38}$. Ce sont les intérêts, matériels et « idéels », et non les idées, qui gouvernent directement la conduite 
humaine. Mais les images du monde suscitées par les idées ont très souvent déterminé, en jouant le rôle d'aiguillages, les voies sur lesquelles la dynamique des intérêts a fait avancer le comportement humain ${ }^{39}$. Dans «l'énorme enchevêtrement des influences réciproques $»^{40}$, Max Weber a certes attaché plus d'importance aux facteurs spirituels qu'au conditionnement socio-économique. Mais ceci relève du caractère arbitraire de toute sélection; il s'agit là d'une démarche heuristique et non point de croyance en la détermination, en dernière instance, par les idées religieuses. Max Weber veut étudier la part qui revient au facteur religieux dans le développement des différentes civilisations, ainsi que la façon dont il a été lui-même influencé, « dans son caractère et son devenir, par l'ensemble des conditions sociales, en particulier par les conditions économiques ${ }^{41}$. Il ne cherche pas les rapports de causalité dans un seul sens. Il explique d'abord les religions par la situation des classes qui les ont créées ou reçues, puis il suit les conséquences de la force propre aux idées religieuses. Il tient à souligner «que les idées religieuses ne se laissent pas déduire tout simplement des conditions économiques...; elles portent en elles la loi de leur développement et possèdent une force contraignante qui leur est propre $»^{42}$. Il étudie la logique originelle, "à la fois idéologique et affective ", d'un système religieux, et les transformations que celui-ci subit, lors des restructurations successives qu'opèrent les différentes générations de fidèles et les différentes couches sociales. "Loin de postuler une action directe des doctrines théologiques sur le comportement économique, la thèse wébérienne suppose l'existence de médiations indispensables, en particulier de motivations psychologiques découlant des croyances religieuses $»^{43}$. Max Weber affirme que jamais l'éthique économique ne fut déterminée par la seule religion; la détermination religieuse de la conduite de la vie n'est qu'une variable parmi d'autres et elle est elle-même profondément influencée par des facteurs économiques et politiques. C'est ainsi qu'il souligne la dépendance initiale d'une religion donnée par rapport à une certaine situation de classe et montre comment, une fois constitué, le système religieux peut faire sentir son influence bien au-delà de la couche sociale qui l'a vu naître.

13 W. Sombart fut, au sein du Verein für Sozialpolitik, l'admirateur le plus enthousiaste de K. Marx. En 1902 encore, dans la première édition de Der moderne Kapitalismus, il écrit que ce qui le sépare de G. Schmoller, c'est le postulat d'une explication en dernière instance, qui est valable pour tous les phénomènes historiques, et leur analyse en termes de système social, «bref, ce que j'appelle le cadre spécifiquement conceptuel. Je pourrais également dire que, ce qui nous sépare, c'est Karl Marx $»^{44}$. W. Sombart remettra en cause progressivement son adhésion, tempérée certes, au matérialisme historique. Dans Le bourgeois ${ }^{45}$, il examine la thèse selon laquelle « les exigences philosophiques et religieuses, loin d'avoir donné naissance à l'esprit capitaliste, ne seraient que le reflet de certaines conditions économiques qui trouveraient leur expression dans une certaine mentalité économique ». Il reconnaît que quel que soit le génie de son fondateur, une religion ne peut prendre racine que si certaines conditions préalables se trouvent remplies dans le milieu extérieur. Mais, selon lui, ces conditions préalables ne sont pas seulement de nature économique, elles sont également d'ordre biologique et ethnologique. "L'acceptation d'une religion (ou, dans une moindre mesure, d'une philosophie) dépend de l'état général d'un peuple, de la composition de son sang, de ses conditions sociales. Pour qu'une religion s'implante et se développe dans une certaine direction, il faut que le peuple possède une certaine disposition. Pas plus qu'une graine ne peut germer sur un rocher nu, une religion humanitaire et philosophique ne saurait s'imposer à des sauvages ignorants et grossiers $»^{46}$. Les problèmes économiques ont pris progressivement une place 
prépondérante dans la sphère d'intérêt de l'homme occidental. W. Sombart constate qu'une religion « subit l'influence des conditions économiques dans une mesure d'autant plus grande qu'elle est plus récente ». S'écartant quelque peu de la thèse wébérienne, il affirme que la formation des différents courants calvinistes du protestantisme se ressent fort nettement de l'influence de l'économie capitaliste avancée, et que si le puritanisme a fini par reconnaître que le genre de vie bourgeois était compatible avec l'état de grâce, cela s'est fait sous l'influence et sous la pression des conditions économiques. «Nous savons à quel point il était, de par sa nature, étranger au capitalisme. Les prédicateurs puritains des $\mathrm{XVI}^{\mathrm{e}}$ et $\mathrm{XVII}^{\mathrm{e}}$ siècles auraient fort volontiers envoyé à tous les diables le culte de Mammon, pour le remplacer par une organisation économique plus appropriée à leurs doctrines anti-mondaines. Mais il était trop tard. Il leur fut impossible de fermer les yeux sur les progrès du capitalisme, comme l'a fait le luthérianisme dans l'Allemagne d'alors, plongée dans la plus profonde misère économique ${ }^{47}$. Il n'en veut pour preuve que le fait que, pour exposer leurs idées sur le salut, les Puritains eurent recours à des représentations et des images empruntées à la vie économique. "C'est ainsi qu'ils conçoivent le saint comme tenant une comptabilité de ses péchés, comme faisant une distinction entre capital et intérêts, ce qui donne à la sanctification de la vie le caractère d'une simple affaire commerciale $\aleph^{48}$. Par ailleurs, pour W. Sombart, l'accumulation des richesses ne fut pas moins décisive pour la naissance du capitalisme que «les forces morales ». "Jamais et en aucun cas des aspirations purement morales ne peuvent engendrer des formes économiques. Il s'agit là d'un malentendu contre lequel Max Weber avait déjà protesté avec force lorsqu'on l'avait accusé d'avoir voulu déduire le capitalisme tout entier de motifs religieux $»^{49}$.

14 Réciproquement, W. Sombart souligne l'influence décisive que les idéologies religieuses exercent sur la vie économique. A partir du moment où un système religieux est bien enraciné, « les doctrines dont il se compose et qu'illumine l'auréole du supra-sensible agissent à leur tour sur la vie en général, sur la vie économique en particulier. Et il serait même étonnant que l'état psychique des sujets économiques échappât à l'influence de ces commandements moraux systématiquement élaborés et impérativement proclamés ${ }^{50}$. Il reconnaît que les idées religieuses ont joué un rôle important dans la constitution de l'esprit capitaliste. Cependant, cette influence a considérablement diminué lorsque le système économique fut solidement établi.» ... Tant qu'un système économique est encore en voie d'édification, tant que chacun est libre d'adopter la conduite économique qui lui plaît, les doctrines morales et les maximes morales qui en découlent ont, pour se déployer, un champ beaucoup plus vaste qu'à l'époque où les différentes branches du système économique ont atteint leur plein et complet développement, où tous les procédés et toutes les démarches sont, pour ainsi dire, mécanisés, où les sujets économiques sont poussés, malgré eux, à adopter une ligne de conduite déterminée ${ }^{51}$.

\section{L'esprit du capitalisme}

15 Ce n'est qu'en 1880 qu'est apparu le mot « capitalisme »; les milieux socialistes allemands furent les premiers à l'utiliser. Il est, dit Lucien Febvre, made in Germany. « C'est un de ces mots en « ismus » caractéristique de la fin du XIX $x^{e}$ siècle, un de ces mots qui répondent à la notion allemande du Wesen, de la qualité essentielle des choses $»^{52}$. Après les socialistes (notamment Mehring et Engels), ce furent les économistes et les capitalistes qui s'en emparèrent. Il passa ensuite aux historiens et aux sociologues, et, dès 1902, W. Sombart 
"l'arbore triomphalement" sur la couverture de la première version de Der moderne Kapitalismus. En 1904, Jacob Strieder publie Zur Genesis des modernen Kapitalismus et Max Weber Die protestantische Ethik und der Geist des Kapitalismus. Max Weber et Werner Sombart furent ainsi parmi les premiers à utiliser le terme de capitalisme dans l'enceinte universitaire et à entreprendre des travaux relatifs à ce concept. Mais l'une des causes fondamentales de la controverse qui les oppose, quant aux origines du capitalisme et au rôle que les juifs ont joué dans son essor, réside dans le fait qu'ils n'utilisent pas la même définition du phénomène.

Dans l'acception vulgaire du terme, le capitalisme désigne toute manifestation d'échange économique, d'usage monétaire et de poursuite lucrative d'un gain. Tout ce qui manifeste un quelconque effort d'enrichissement, que ce soit par la conquête militaire, par l'expropriation politique, par le vol ou l'escroquerie, est désigné par le vocable capitalisme. M. Weber récuse une définition aussi large et hétéroclite. Selon lui, le concept de capitalisme doit être composé graduellement, à partir de ses éléments singuliers qui sont à extraire un à un de la réalité historique. C'est donc, au cours de la recherche que le concept se précisera progressivement, "selon les points de vue» arbitrairement retenus à l'origine. «En outre, ces points de vue à partir desquels les phénomènes historiques que nous étudions peuvent être analysés, ne sont en aucune manière les seuls possibles. Ainsi qu'il en va pour chaque phénomène historique, d'autres points de vue nous feraient apparaître d'autres traits comme essentiels. Il s'ensuit, sans plus, que sous le concept d'esprit du capitalisme il n'est nullement nécessaire de comprendre seulement ce qui se présente à nous comme essentiel pour l'objet de nos recherches. Cela découle de la nature même de la conceptualisation des phénomènes historiques (historische Begriffsbildung), laquelle n'enchâsse pas, à toutes fins méthodologiques, la réalité dans des catégories abstraites, mais s'efforce de l'articuler dans des relations génétiques concrètes qui revêtent inévitablement un caractère individuel propre $»^{53}$.

\section{Les origines et les étapes du capitalisme selon Max Weber}

Pour Max Weber il y a eu, à l'origine du capitalisme moderne, une collusion entre le développement de l'économie et un certain type de calvinisme. L'irruption de l'esprit capitaliste a coïncidé avec les dispositions prises « pour garantir, de manière nouvelle et positive dans le contexte d'une conception neuve de la récompense religieuse, un gain à toute activité menée avec méthode et conscience pour acquérir les biens de ce monde " (B. Nelson). Ce que Luther et Calvin ont introduit, c'est un changement radical quant aux éléments rationnels de la conscience morale, qui est le centre de direction de toutes les matrices de décision affectant la croyance et la conduite, chez l'individu et dans le groupe. Tant que les valeurs suprêmes de la société occidentale ont été des valeurs nobiliaires et ecclésiastiques, l'homme voué à accumuler des gains n'avait qu'une activité illégitime. Le but suprême du bourgeois, ainsi que le souligne Jean Baechler ${ }^{54}$, était d'accéder à un mode de vie nobiliaire, caractérisé par la possession d'une terre et de rentes, c'est-à-dire de renoncer à son activité économique. « Là où, au contraire, l'activité économique s'est trouvée valorisée pour elle-même, la bourgeoisie a connu un développement bien plus intense ». Reprenant l'analyse de Max Weber, Jean Baechler affirme que l'éthique protestante n'a pas agi tellement en conférant à l'activité économique sa rationalité, qu'en donnant aux bourgeois le sentiment que leur mode de 
vie était légitime. Elle leur a permis de recouvrer leur dignité, sans renoncer à leur activité économique.

Le capitalisme, comme tout phénomène historique, ne s'est pas développé selon une progression continue et harmonieuse, mais en une suite de tensions, de ruptures, d'accélérations et de ralentissements. L'éthique protestante et l'esprit du capitalisme analyse un moment essentiel du processus de rationalisation, qui caractérise la civilisation occidentale. Chez les moines médiévaux on perçoit déjà nettement cette rationalisation fondée sur une ascèse de la vie. «L'ascèse puritaine n'a donc aucune vertu inchoative, car elle n'est que la reprise, sous une autre forme et dans d'autres conditions historiques, de l'ascèse monacale. Weber n'a donc jamais nié l'existence d'un capitalisme bancaire dans les villes du sud, mais il insiste surtout sur le phénomène nouveau du capitalisme d'entreprise qui apparaît plus d'un siècle après la Réforme $»^{55}$. Une des caractéristiques de ce nouveau capitalisme consiste en l'application du rationalisme ascétique à l'économie. Pour Max Weber, toute pensée ou activité rationnelle peut contribuer à l'émergence du système capitaliste, dès qu'elle s'applique au domaine économique, même lorsque l'économie n'est pas envisagée comme fin en soi. Il relève le rôle des moines, pas seulement en Occident, mais aussi au Tibet, au Japon, en Chine, partout où des communautés fortement structurées ont à gérer un patrimoine ou à assurer leur subsistance. "A cette époque, le moine est le premier être humain qui vit rationnellement, qui travaille méthodiquement et par des moyens rationnels à poursuivre un but, à savoir l'au-delà... La vie économique des communautés monastiques était rationnelle $\aleph^{56}$. Max Weber est parfaitement conscient du fait que le capitalisme a préexisté au protestantisme. Il a mené de nombreuses recherches sur le capitalisme antique, d'abord dans sa Habilitationsschrift de 1891 sur Die römische Agrargeschichte in ihrer Bedeutung für das Staats-und Privatrecht, ensuite dans deux études ultérieures, l'une de 1896 Die sozialen Gründe des Untergangs der Antiken Kultur, l'autre de 1897, Agrarverhältnisse im Altertum. Tous ces écrits sont antérieurs à L'éthique protestante et lui ont permis de saisir le phénomène capitaliste dans son ensemble, avant d'évoquer le nouveau visage qu'il a pris durant le $\mathrm{XVII}^{\mathrm{e}}$ siècle. Le capitalisme n'est pas né à cette époque, car il existait déjà sous des formes plus ou moins élaborées, plus ou moins rationnelles à la fin de l'Antiquité et au Moyen Age. Le capitalisme moderne représente, cependant, un phénomène radicalement neuf, qui frappe par sa singularité. "En général, cette révolution ne dépend pas d'un afflux d'argent frais - je connais des cas où il a suffi de quelques milliers de marks empruntés à des parents - mais d'un "esprit nouveau »: l'esprit du capitalisme est entré en action. Le problème majeur de l'expansion du capitalisme moderne n'est pas celui de l'origine du capital, c'est celui du développement de l'esprit du capitalisme. Partout où il s'épanouit, partout où il est capable d'agir luimême, il "crée» son propre capital et ses réserves monétaires-ses moyens d'action- mais l'inverse n'est pas vrai $\aleph^{57}$. Max Weber a souligné le caractère dramatique de l'histoire, en montrant comment, paradoxalement, les conséquences trahissent les intentions des hommes. C'est ainsi que les protestants ont accumulé les richesses qu'ils condamnaient, que les moines ont, par ascétisme, créé ce qu'ils refusaient. Les juifs, qui voulaient soumettre leur activité économique à l'impératif d'éthique sociale de la berith, ont parfois été conduits à assumer le double rôle d'exploité et d'exploiteur.

Pour Max Weber, il y a une adéquation significative entre l'esprit du capitalisme et l'esprit d'un certain protestantisme, une affinité spirituelle entre une vision du monde et un certain style d'activité économique. Il n'a pas considéré le calvinisme originel ou 
orthodoxe, mais la mise en pratique, au prix peut-être de déformations, d'une éthique que certaines couches calvinistes ont intériorisée à leur manière. Il reconnaît que le calvinisme qu'il étudie est même contraire à l'authentique doctrine de Calvin. «Ce n'est donc pas la doctrine en elle-même, mais bien les conséquences psychologiques d'une certaine interprétation du dogme sur la conduite pratique de la vie qui intéressent Weber $\aleph^{58}$. Pour fonder sa théorie du rôle éminent du protestantisme à l'origine du capitalisme, il s'emploie à montrer que les autres antécédents du système, tels que les réserves d'or, les découvertes, les techniques, le droit et l'organisation politique n'ont pas suffi à susciter, ailleurs, le capitalisme. Parmi les obstacles que rencontra dans les autres cultures le développement du capitalisme, il faut, selon Max Weber, citer, au même titre que certains traits de l'organisation politique, les croyances religieuses. «Même le confucianisme, bien qu'il fût soucieux avant tout de la vie ici-bas, rationnel et économique, ne poussait pas au travail d'entreprise. Bien plus, par le traditionalisme, par la modération qu'il enseignait, il était opposé à une économie capitaliste $»^{59}$. Weber ne s'intéresse nullement au développement du capitalisme en général, mais à l'esprit particulier et à l'ethos singulier des entrepreneurs qui sont à l'origine du capitalisme moderne. Il a donc circonscrit aussi clairement que possible son problème.

Max Weber reconnaît explicitement que le capitalisme moderne de la société industrielle du XIX ${ }^{e}$ siècle a perdu son esprit originel, en ce sens que les entrepreneurs ont abandonné l'ethos particulier de leurs prédécesseurs. Une fois le système capitaliste solidement implanté, il fonctionne en vertu de sa propre dynamique. Il n'est plus besoin, alors, de motivation métaphysique ou morale pour que les individus se conforment à la loi du capitalisme ; celle-ci s'impose à eux. Max Weber souligne qu'à l'époque contemporaine, avec nos institutions politiques, légales et économiques, avec la structure et les formes d'organisation générales, propres à notre ordre économique, l'esprit du capitalisme peut apparaître purement et simplement comme le résultat d'une adaptation. Le système capitaliste a besoin tout au plus du dévouement à la vocation de gagner de l'argent. "Cette attitude à l'égard des biens matériels est à ce point adaptée au système, si intimement liée aux conditions de survie dans la lutte économique pour l'existence, qu'il ne saurait plus être question, aujourd'hui, d'une relation nécessaire de cette façon de vivre avec une quelconque Weltanschauung moniste. En fait, ceux qui adoptent cette attitude n'ont plus besoin du soutien d'aucune force religieuse, et ils ressentent les tentatives de la religion pour influer sur la vie économique-dans la mesure où ces tentatives sont encore sensibles- comme des entraves analogues à la réglementation de l'économie par l'État». Ce sont les intérêts commerciaux, sociaux et politiques qui tendent à déterminer opinions et comportements. Quiconque n'adapte pas sa conduite aux conditions du succès capitaliste va à sa perte ou, à tout le moins, ne peut s'élever bien haut. «Ces phénomènes sont ceux d'une époque où le capital moderne, ayant remporté la victoire, s'est émancipé de ses anciens tuteurs $\aleph^{60}$. Le capitalisme est devenu, par sa propre dynamique, le système économique général de la société occidentale, " indépendamment de toute pesanteur morale, religieuse et politique, c'est-à-dire qu'il s'est libéré de la tutelle des autres activités sans briser, cependant, toutes les relations » (J. Freund).

21 Pour Raymond Aron ${ }^{61}$, la définition wébérienne du capitalisme, dont la caractéristique essentielle est l'entreprise travaillant en vue de l'accumulation indéfinie du profit et fonctionnant selon une rationalité bureaucratique, est à la fois proche de celle de Marx, et s'en écarte sur plusieurs points. Comme ce dernier, Max Weber pense que l'essence du 
régime capitaliste est la quête du profit par l'intermédiaire d'un marché régulier. Lui aussi insiste sur la présence des travailleurs juridiquement libres, louant leur force de travail au propriétaire des moyens de production; il souligne, enfin, que l'entreprise capitaliste moderne utilise des moyens de plus en plus puissants, renouvelant perpétuellement les techniques en vue d'accumuler des profits supplémentaires. Le progrès technique résulte d'ailleurs de la concurrence des producteurs. Cependant, à la différence de Marx, Weber estime que la caractéristique essentielle de la société moderne, c'est la rationalisation bureaucratique et, quel que soit le statut de propriété des moyens de production, aucune collectivité ne peut y renoncer. «Max Weber évoquait volontiers une socialisation de l'économie mais n'y voyait pas une transformation fondamentale. La nécessité de l'organisation rationnelle pour obtenir la production au meilleur coût subsisterait au-delà de la révolution qui aurait donné à l'État la propriété des instruments de production $»^{62}$. Le socialisme planificateur, qui est contraint de renforcer le contrôle de l'économie, se situe dans la lignée du capitalisme.

\section{Sources et développement du capitalisme selon W. Sombart}

Werner Sombart utilise des définitions différentes, voire antithétiques du capitalisme. Dans Die deutsche Volkswirtschaft im neunzehnten Jahrhundert, ouvrage publié pour la première fois à Berlin en 1903, il met en avant la recherche d'un profit illimité, l'esprit d'entreprise qui amène l'abandon de l'activité artisanale au profit d'une carrière stimulante et risquée ${ }^{63}$. Cette définition semble s'opposer radicalement à celle que $\mathrm{M}$. Weber a proposée l'année suivante ; elle s'en rapproche, cependant, par certains aspects ; en effet, $W$. Sombart mentionne également la rationalisation de l'entreprise, le refus de la jouissance (Vergnügen) et le sens du devoir (Pflicht ${ }^{64}$. D'une manière générale, il définit le capitalisme par le principe d'acquisition, par opposition à une économie domestique ou naturelle, qui tend à la simple satisfaction des besoins. «L'économie capitaliste est fondée sur l'argent, les échanges, la circulation des richesses, la concurrence ; elle est animée par le désir individuel d'un profit illimité. Il n'est donc pas étonnant que Sombart découvre des formes multiples et successives de capitalisme : capitalisme primitif, haut capitalisme, capitalisme tardif, capitalisme commercial, financier, industriel... ${ }^{65}$. Le capitalisme s'oppose à une économie qui se limite, avant tout, à la satisfaction des besoins ( Bedarfswirtschaft). "Le capitalisme serait le système qu'anime un désir de gains sans limite, qui se développe et progresse sans terme, économie d'échanges et d'argent, de mobilisation et de circulation des richesses, de calcul rationnel. Les caractères sont moins dégagés par une comparaison avec les autres civilisations, que saisis intuitivement dans leur ensemble $»^{66}$. Sombart définit l'esprit du capitalisme d'une manière radicalement opposée à celle de Weber, quand il écrit que le puritanisme n'est pas «responsable de l'épanouissement illimité de l'amour du gain, de la passion insensée de l'argent, de l'idiosyncrasie des affaires, toutes qualités caractéristiques de l'esprit capitaliste à son apogée... La morale puritaine n'a pas favorisé la course éhontée, sans scrupule dans le choix des moyens, aux richesses. Comme la morale thomiste, elle ne se lasse pas de recommander et de prêcher l'emploi de moyens honnêtes ${ }^{67}$. C'est sous la pression des conditions économiques et "à contre-cœur» que les prédicateurs puritains durent s'efforcer de concilier les progrès du capitalisme avec leurs conceptions religieuses et qu'ils finirent par « reconnaître que le genre de vie bourgeois était compatible avec l'état de grâce $»^{68}$. Le capitalisme s'oppose, pour W. Sombart, à la mentalité économique traditionnelle, qui se définit par l'idéal de la subsistance, l'organisation corporative et la 
stabilité. Il introduit une conception essentiellement individualiste de l'économie, conception d'après laquelle la sphère d'action de chaque sujet économique ne devait être limitée par aucune réglementation restrictive portant soit sur le volume de la vente, soit sur la spécialisation et sur la séparation des professions. Selon l'éthique capitaliste, chaque sujet a, au contraire, le droit d'étendre son champ d'action, dans la mesure de ses moyens, même aux dépens de ses voisins. «Les moyens de lutte préconisés tacitement par cette conception étaient essentiellement d'ordre spirituel: ruse, astuce, finesse; les seules limitations dont elle recommandait le respect dans la concurrence économique étaient celles imposées par le code pénal, et, à la condition d'être en règle avec celui-ci, chacun devait avoir la liberté d'agir à sa guise, au mieux de ses intérêts " ${ }^{69}$. Dans Les juifs et la vie économique, $\mathrm{W}$. Sombart définit le capitalisme comme l'organisation économique qui comporte la collaboration régulière de deux catégories différentes de la population: les propriétaires des moyens de production, qui remplissent en même temps les fonctions de direction, et les ouvriers voués uniquement au travail et ne possédant rien. Cette organisation est comprise de telle sorte que les représentants du capital, c'est-à-dire de la réserve des biens nécessaires à la mise en oeuvre et au fonctionnement du processus économique, sont les sujets économiques, chargés à la fois d'initiative et de responsabilité : initiative en ce qui concerne le caractère et l'orientation à donner à la vie économique; responsabilité des résultats obtenus à la suite de leur initiative. La force motrice d'un tel système, c'est l'idée de profit, le capital doit tendre vers sa propre multiplication. "Cette force s'impose à chaque entrepreneur capitaliste comme une contrainte objective et oriente sa conduite dans une direction bien déterminée $»^{70}$. Une telle activité économique, qui poursuit systématiquement la réalisation de profits, engendre de ce fait une tendance à l'expansion incessante des entreprises; elle a pour corollaire une subordination consciente et voulue de tous les actes à la méthode éminemment rationnelle, qui préside à l'activité économique. C'est ainsi qu'à l'organisation précapitaliste de l'économie, fondée sur la tradition et sur le principe de repos, succède la rationalisation de l'économie, comportant un système fondé sur le principe de mouvement ${ }^{71}$. Pour W. Sombart, comme pour M. Weber, le rationalisme est l'un des éléments déterminants du capitalisme. D'où la nécessité de fonder toute entreprise rationnelle sur le calcul, d'établir un plan à long terme et de choisir des moyens efficaces pour le réaliser. W. Sombart met l'accent sur la rationalisation et souligne l'importance du calcul rationnel dans la formation de l'esprit du capitalisme, qu'il décrit comme " un mélange singulier d'aspiration passionnée à l'infini et de froide spéculation rationnelle $»^{72}$. Mais, ainsi que le précise P. Besnard ${ }^{73}$, la rationalisation renvoie moins, chez Sombart, à la discipline qu'à la spéculation. La rationalité économique semble parfois se confondre avec la recherche du résultat optimum quel que soit le moyen employé, et l'organisation rationnelle du travail devient une caractéristique secondaire du capitalisme.

W. Sombart considère comme un élément significatif du système capitaliste les différentes façons de faire du commerce que $\mathrm{M}$. Weber qualifiait de «capitalisme de paria ». " On doit bien se rendre compte qu'il y a mille manières de faire du commerce. Par exemple : on peut équiper et armer des vaisseaux, recruter des soldats, conquérir des pays, mettre à la raison les indigènes à l'aide de fusils et de sabres, les dépouiller de leurs biens, charger ceux-ci sur des vaisseaux et les emporter dans la métropole pour les vendre aux enchères au plus offrant. Ou encore, on peut, après avoir, à force de ruse et d'astuce, et à la suite de multiples affronts, réussi à acheter une vieille culotte à un 
chevalier besogneux, la revendre à un brave paysan qui n'a pas su résister à l'éloquence persuasive du marchand. Ou encore: on peut acheter des valeurs en Bourse, en escomptant la hausse ou la baisse $»^{74}$. Ces activités lucratives sont typiquement capitalistes, dans la mesure où elles font converger vers un seul et même but le calcul, la force et la négociation. Le commerçant capitaliste, en effet, doit être à la fois « un calculateur-spéculateur " et "un homme d'affaires, un négociateur». Pour poser un diagnostic économique, il embrasse d'un seul coup d'oeil tous les phénomènes du marché et saisit le lien qui les unit. «Il faut être à même d'établir la valeur relative des différents symptômes, être à même de prévoir les possibilités de l'évolution ultérieure et, surtout d'entrevoir, avec une certitude qu'on ne saurait prendre en défaut, entre cent possibilités la plus avantageuse. Aussi le commerçant doit-il être tout yeux, tout oreilles, d'une sensibilité infinie. Il doit être à l'affût de chevaliers besogneux, d'États nourrissant des projets de guerre, pour leur offrir au moment opportun l'emprunt sauveur; il doit savoir qu'il existe, quelque part, une catégorie d'ouvriers se contentant d'un salaire inférieur de quelques centimes à celui des autres. Ici, il s'agit de bien calculer si tel article nouveau a des chances d'être bien accueilli du public; là il s'agit de bien mesurer l'influence qu'un événement politique donné peut exercer sur les dipositions du marché des valeurs ${ }^{75}$. Dans la poitrine du capitaliste cohabitent deux âmes radicalement différentes, « deux âmes, cependant, qui, à la différence de celles du docteur Faust, loin de chercher à se séparer l'une de l'autre, accomplissent l'oeuvre commune en parfaite harmonie, là où l'entreprise capitaliste a atteint son plus complet développement $»^{76}$. Il s'agit de la réunion en une seule personne de l'entrepreneur et du commerçant, qui fondent leurs deux âmes en une seule dans le sujet de l'économie capitaliste, au point de former une individualité nouvelle, tout à fait particulière. L'entrepreneur est un homme qui se consacre entièrement à la tâche qu'il doit accomplir; chacun de ses actes n'est conçu et accompli qu'en vertu de l'oeuvre qu'il doit réaliser. Le commerçant est un homme qui veut faire des affaires lucratives, « un homme dont toutes les idées et tous les sentiments sont concentrés sur la valeur en argent que représentent les états et les actions les plus variés, un homme, par conséquent, qui évalue en argent tous les phénomènes. Le monde n'est pour lui qu'un grand marché, avec offres et demandes, conjonctures, chances de gain ou de perte $»^{77}$. L'entrepreneur capitaliste est à la fois l'inventeur de nouvelles formes d'organisation économique, tant de la production que de la vente, un conquérant qui renverse tous les obstacles dressés sur son chemin et prend des risques, et un organisateur qui sait faire collaborer un grand nombre d'hommes, afin qu'ils produisent un maximum de biens. Le commerçant capitaliste est un habile négociateur, qui sait convaincre son interlocuteur des avantages d'une affaire. «Fait du commerce, toujours dans ce sens spécifique du mot, le petit marchand ambulant qui discute avec la cuisinière le prix d'une peau de lapin, ou le juif, marchand de vieux habits, qui négocie pendant une heure avec un brave paysan la vente d'un pantalon; ou encore Nathan Rothschild qui, dans une conférence de plusieurs jours, négocie avec l' « intermédiaire » prussien, dans des conditions extraordinairement compliquées, un emprunt de plusieurs millions. Les différences qui existent entre ces trois formes de négociations sont purement quantitatives; mais le fond est le même; ce qui fait l'âme de tout commerce moderne, c'est la négociation... $»^{78}$.

Dans la première édition de Der moderne Kapitalismus (1902), W. Sombart fait débuter le capitalisme en l'année 1204, qui vit la chute de Constantinople et l'introduction des chiffres arabes dans les opérations de calcul. Dans l'édition de 1916, il intègre comme éléments décisifs de la formation du capitalisme, la production des métaux précieux, les 
rentes urbaines, le rôle des Juifs, celui du luxe et de la guerre. Tous ces éléments se combinent en un système par la force de quelque esprit mystérieux, ou encore sous l'effet du hasard. Sombart ne le précise pas. Dans l'édition de 1927, il distingue «l'aube du capitalisme ", qui s'étend de 1500 à 1760 , du « capitalisme à son apogée » qui s'étend de 1760 à 1814. J. Le Goff ${ }^{79}$, discutant la thèse de Sombart, estime qu'il vaut mieux considérer le grand marchand médiéval comme un pré-capitaliste. Selon une définition stricte du capitalisme, comme en offre la doctrine marxiste, le Moyen Age n'a pas connu le capitalisme. Son système économique et social, c'est la féodalité et c'est à l'intérieur de ce cadre qu'agissent les «mercatores ». "Mais ils contribuent à faire éclater ce cadre, à ruiner les structures féodales. En agissant sur une évolution agricole activée par l'intrusion des capitaux urbains -au moins en des régions comme l'Italie ou la Flandre -, précipitée par l'élargissement d'une économie mondiale qui a de profondes répercussions sur les prix agricoles et industriels, les grands marchands préparent l'avènement du capitalisme $»^{80}$. Jacques Le Goff ${ }^{81}$ rappelle à juste titre qu'il ne faut pas oulier que l'économie médiévale reste fondamentalement rurale, que dans les villes l'artisanat prédomine, que les grandes affaires ne sont qu'une couche superficielle. "Mais par la masse d'argent qu'il manie, par l'étendue de ses horizons géographiques et économiques, par ses méthodes commerciales et financières, le marchand-banquier médiéval est un capitaliste. Il l'est aussi par son esprit, son genre de vie, par sa place dans la société ».

Dans Les juifs et la vie économique, Sombart va jusqu'à associer le capitalisme et l'économie pastorale, faisant du premier le prolongement de l'activité des bergers nomades ${ }^{82}$. Les différences entre l'économie de subsistance et l'économie capitaliste relèvent de l'opposition entre le «saharisme » et le «sylvanisme ». C'est de la forêt défrichée et de la terre labourée qu'est née l'organisation "paysanne-corporative» ou «féodalecorporative ", fondée sur le principe de la subsistance, du travail exécuté, et sur la division en classes sociales. « C'est le patrimoine délimité du paysan qui fait naître l'idée d'un cercle d'activité également délimité, dans lequel chaque sujet économique se trouve confiné une fois pour toutes, et dans lequel il se comporte d'une manière qui reste invariable pendant des siècles (traditionalisme); c'est de là que l'idée de la subsistance s'étend à toutes les autres branches d'activité, en les façonnant à l'image de l'activité rurale. Au-dessus de ces unités économiques, représentant chacune une réalisation différente du principe de la subsistance, mais reliées les unes aux autres, en fait d'abord, en droit ensuite, s'édifie la construction organique de l'État composé de classes ${ }^{83}$. Par contre, c'est du désert infini et de l'économie pastorale, que naît le capitalisme. Son champ d'activité, c'est l'étendue illimitée où paissent les troupeaux ; sa spécificité, c'est la spéculation et le risque, car le cheptel peut augmenter rapidement ou bien être décimé. "C'est seulement ici, dans l'économie pastorale, jamais dans la sphère de l'agriculture, que l'idée du gain a pu prendre racine. C'est ici seulement que l'économie était fondée sur le principe de la multiplication indéfinie de la quantité des produits... Ici seulement a pu naître l'idée que c'est la quantité abstraite de biens, et non la qualité d'usage, qui forme la principale catégorie de la vie économique. C'est ici que le calcul et le dénombrement ont été pour la première fois introduits dans l'économie. Mais les éléments rationnels qui ont pénétré dans la vie économique viennent, eux aussi, du nomadisme, qui peut ainsi être considéré à tous égards comme le père du capitalisme. Et nous voyons de nouveau, mais plus clairement qu'auparavant, la manière dont le capitalisme se rattache au judaïsme, celui-ci servant de pont intermédiaire entre celui-là et son prototype, le nomadisme $»^{84}$. 
Dans Le bourgeois, Sombart affirme que c'est dans le commerce que l'esprit capitaliste a pris son essor, car le négoce contraint l'homme à se préoccuper uniquement de l'aspect quantitatif des choses. Tandis que le producteur précapitaliste, qu'il soit paysan ou artisan, est toujours préoccupé par la qualité, et ne voit dans les objets qu'il produit que des biens de consommation qualitativement différents, le marchand renonce à l'évaluation qualitative, car aucun lien organique ne le rattache aux biens dont il fait commerce. "Le paysan ou l'artisan se confond, jusqu'à un certain point, avec chaque objet qu'il produit, il se retrouve lui-même dans chaque-objet, dans lequel il a mis un peu de son âme, et auquel il se trouve rattaché par des liens intimes $»^{85}$. Le marchand, au contraire, ne voit dans les objets de son commerce que des objets d'échange, qu'il évalue quantitativement, selon leur valeur en argent. De plus, le commerce international crée l'habitude de vivre et de penser rationnellement, de changer facilement de résidence et de milieu. Enfin, le prêt d'argent, où tout ce qui se rattache à la qualité et au principe de la subsistance se trouve éliminé; a "puissamment contribué au développement du capitalisme $»^{86}$. Ce qui est déterminant dans la formation du capitalisme, c'est das Erwachen des Erwerbstriebes («l'éveil de la soif du profit»). Cet esprit nouveau, qui se définit par l'attrait du gain, le sens du calcul et le rationalisme économique, naît dans la mentalité de l'entrepreneur. W. Sombart évoque « une espèce de nécessité psychique » ( eine Art von psychischem Zwang), qui contraint l'entrepreneur à accumuler toujours davantage. Voulant analyser, comme M. Weber, les " premières phases du capitalisme ", il affirme que ce sont les Juifs qui ont brisé les cadres de la vieille morale économique, pour se lancer à la poursuite du gain, "sans tenir compte d'aucune limitation, sans $s^{\prime}$ embarrasser d'aucun scrupule ${ }^{87}$. Les éléments constitutifs de la morale économique nouvelle ont eu chez les Juifs « une avance de dix siècles $»^{88}$. Durant la première période du capitalisme, la mentalité économique juive se caractérisait par sa modernité : par contraste avec la mentalité dominante, elle représentait une "conception essentiellement individualiste de l'économie» et affirmait les principes du "libre commerce » et de la « libre concurrence $»^{89}$.

Prenant le contre-pied de Weber, Sombart établit la responsabilité partielle de certains courants du catholicisme, tel le thomisme, dans l'apparition du capitalisme, et minimise considérablement celle du protestantisme. Pour faire ressortir l'influence que le catholicisme a exercée sur la formation de l'esprit capitaliste, $\mathrm{W}$. Sombart se propose d'analyser le thomisme non pas en tant que système théologique, mais en tant qu'il détermine une certaine attitude face à la vie économique et qu'il modèle la conduite quotidienne des fidèles. C'est précisément dans cette optique que Max Weber avait étudié les rapports du puritanisme et de la vie économique. L'idée fondamentale de la morale thomiste consiste dans la rationalisation de la vie : «le rationalisme économique n'aurait jamais acquis la rigidité et subi l'intériorisation que nous lui connaissons, sans le secours d'un système de règles rationnelles, achevé dans tous ses détails, et s'appuyant sur la suprême autorité de l'Église. L'effet de ces doctrines sur la mentalité économique devait être particulièrement profond lorsqu'elles réussissaient (comme ce fut le cas) à engendrer des états psychiques qui, par leur nature, étaient favorables à l'esprit capitaliste $»^{90}$. Et W. Sombart de mentionner la condamnation de la jouissance et de l'oisiveté, ainsi que la valeur attachée à l'honnêteté. De tels préceptes «visaient à une sorte de dressage psychique $»^{91}$. Mais il s'empresse d'ajouter que les « vertus bourgeoises » qu'a enseignées l'éthique thomiste n'ont rien à voir avec l'esprit d'individualisme économique, dont il tient le judaïsme pour responsable. Le protestantisme qui, selon W. Sombart ${ }^{92}$, prône un 
idéal de pauvreté et dénonce l'amour de l'argent comme la source de tous les maux, qui méprise les biens terrestres et exalte la communion avec Dieu, constitue un obstacle au développement de l'esprit du capitalisme. Progressivement, cependant, le puritanisme s'est approprié les principes de l'éthique thomiste, « en apportant à leur défense autant d'ardeur passionnée que d'étroitesse d'esprit ». A son tour, la morale puritaine affirme la nécessité de la rationalisation et de la «méthodisation » de la vie et des instincts, « de la transformation de l'homme naturel en un homme rationnel». W. Sombart se rallie à l'analyse de Max Weber ${ }^{93}$, pour reconnaître que «les variétés non-luthériennes du protestantisme » ont amené chacun de leurs membres à prendre conscience de «la nécessité d'un contrôle méthodique de sa conduite ». Lorsque la morale puritaine exhorte les fidèles à mener une vie bien ordonnée, elle ne fait, selon W. Sombart, que reproduire mot pour mot les préceptes de la morale thomiste, et les vertus bourgeoises qu'elle prêche sont exactement les mêmes que celles dont on trouve l'éloge chez les scolastiques: l'application au travail, la modération et l'esprit d'épargne. La morale puritaine a même poussé l'esprit d'épargne jusqu'à la "mesquinerie ». Cependant, le capitalisme n'est pas uniquement le produit de l'éthique protestante. «En premier lieu, les vertus bourgeoises existaient déjà deux cents ans environ lorsque le puritanisme est né. Elles sont déjà décrites et exposées avec tous les détails désirables dans les livres de famille d'Alberti. S'il est un système religieux qui ait pu favoriser leur éclosion, ce ne pouvait être que le catholicisme. La morale protestante n'a eu qu'à s'approprier ce que le thomisme a créé $»^{94}$. Par ailleurs, selon W. Sombart, le puritanisme n'est pas responsable de l'épanouissement illimité de l'amour du gain, de la passion insensée de l'argent et de l'idiosyncrasie des affaires, qui caractérisent l'esprit capitaliste à son apogée. C'est à son corps défendant qu'il admet le désir d'enrichissement et toujours avec cette limitation tacite ou explicite : le gain et l'enrichissement ne constituent pas des fins en soi. D'autre part, W. Sombart souligne que la morale puritaine n'a pas favorisé la course aux richesses éhontée, sans scrupule dans le choix des moyens, et que, comme la morale thomiste, elle ne se lasse pas de recommander et de prêcher l'emploi de moyens honnêtes. Relevons ici une contradiction dans l'analyse sombartienne, puisque dans un autre passage du Bourgeois, il définit précisément l'honnêteté et la loyauté commerciale, par intérêt bien compris, comme l'une des principales caractéristiques du capitalisme. En dernier lieu, il affirme qu'il est faux d'établir un rapport de cause à effet entre la conception du monde puritain et le grand épanouissement de l'esprit d'entreprise, à l'époque post-puritaine, dans les pays à population de souche puritaine. «Le puritanisme était loin d'encourager ses adeptes à se lancer dans des entreprises de vaste envergure ou aventureuses: il se contentait de les voir faire preuve d'une mentalité de boutiquiers sages et terre-à-terre » ${ }^{95}$. Ainsi, l'éthique puritaine ne constitue que l'un des facteurs ayant exercé une influence sur le développement de l'esprit capitaliste, et encore s'agit-il d'« une influence minime » ${ }^{96}$.

\section{La controverse entre Max Weber et Werner Sombart : un faux débat}

La divergence fondamentale entre la définition wébérienne et la définition sombartienne du capitalisme invalide à l'avance toute confrontation sérieuse. D'autant plus que la définition mise en avant par W. Sombart est des plus fluctuantes. L'analyse wébérienne a bien résisté aux multiples assauts qu'on a livrés contre elle, souvent avec beaucoup de mauvaise foi, en s'abstenant de lire sérieusement l'oeuvre de Max Weber. H. M. Robertson ${ }^{97}$ situe, tout comme W. Sombart, au début du XIII ${ }^{\mathrm{e}}$ siècle les premières manifestations, 
tant matérielles qu'intellectuelles, du capitalisme. En 1202, Pisano publie le Liber Abbaci qui pose les principes du calcul commercial, dont l'emploi se généralise en Italie à partir du XIV ${ }^{e}$ siècle. La même année débute la quatrième Croisade dont l'aboutissement, la prise de Constantinople par les Croisés en 1294, inaugure l'ère de l'hégémonie commerciale de Venise et amorce l'exploitation économique de l'Orient par l'Europe occidentale. Il mentionne encore de nombreuses manifestations du capitalisme antérieures à la Réforme. Or, que le Moyen Age ait connu certaines formes de capitalisme, personne n'en disconviendra. Mais, rétorquerons-nous avec Philippe Besnard ${ }^{98}$, s'il est facile de rechercher et de découvrir dans un passé lointain des faits capitalistes, encore faut-il se demander si ces faits constituent un système, s'ils ne sont pas isolés et fragmentaires. De plus, Weber n'a jamais prétendu que le capitalisme était né au XVI ${ }^{e}$ siècle, à Wittenberg ou à Genève. C'est précisément contre une telle déformation de ses intentions qu'il avait protesté à l'avance, dès 1904: "Il est hors de question de soutenir une thèse aussi déraisonnable et doctrinaire, qui prétendrait que l'esprit du capitalisme (...) ne saurait être que le résultat de certaines influences de la Réforme, jusqu'à affirmer que le capitalisme en tant que système économique est une création de celle-ci. Le fait que telle ou telle forme d'organisation capitaliste soit considérablement plus ancienne que la Réforme en est une réfutation suffisante ». M. Weber est le premier à reconnaître qu'il y a eu, dans de nombreux pays, « un capitalisme et des entreprises capitalistes reposant sur une rationalisation passable des évaluations en capital $»^{99}$. Ce qu'il entend étudier, c'est le capitalisme moderne, qui porte l'empreinte de l'éthique protestante. Par ailleurs, M. Weber ne nie pas l'importance de facteurs très divers qui ont favorisé l'essor du capitalisme moderne, tels les grandes découvertes, le développement d'un droit et d'une technique rationnels, et en particulier la comptabilité scientifique. Il n'entendait pas annuler les explications données jusqu'alors de l'essor du capitalisme mais attirer l'attention sur un point particulier. "Sa démarche, qui isole certains éléments caractéristiques dans la réalité historique, n'est-elle pas plus fructueuse -à condition que l'on ne soit pas dupe du procédé- que celle de Sombart, qui dans Le bourgeois, présente un véritable catalogue des causes possibles de l'essor de l'esprit capitaliste? A s'en tenir aux seuls titres de chapitres, l'énumération est impressionnante : elle va de la passion de l'or et de l'amour de l'argent aux conditions techniques et politiques, en passant par les vertus bourgeoises, l'emploi du calcul, les prédispositions ethniques, les migrations et les forces morales (philosophie et influences religieuses). Dans l'essai sur L'éthique protestante, Weber privilégie un aspect particulier de la réalité, il ne nie pas pour autant, nous l'avons $\mathrm{vu}$, la multiplicité des influences ni leur réciprocité $\aleph^{100}$. Certes, l'éthique du travail et de la doctrine des vertus bourgeoises, que Weber attribue notamment à Benjamin Franklin, se trouvaient déjà exprimées au Quattrocento dans les écrits de Léon Battista Alberti. La rationalisation de la conduite économique, l'esprit d'épargne, la sobriété, la loyauté commerciale, l'honorabilité, telles sont les principales vertus que prônent Alberti et d'autres hommes de son époque. En quatre siècles, affirme Sombart, "pas un trait du tableau n'a subi de modification ». Cependant, les Anciens, tels Caton et Marc Aurèle, et les lettrés de la Renaissance, tel Alberti, s'adressent à une élite, et se préoccupent « de la sagesse dans la conduite de la vie et non pas d'éthique ». Il n'y a nulle trace chez eux de cette « résonance morale pathétique » qui fait tout le caractère du sermon aux jeunes marchands, de Franklin ${ }^{101}$. A ces « sagesses temporelles », au « rationalisme » économique des auteurs de l'Antiquité ou d'Alberti, il manque par-dessus tout «une force révolutionnaire comparable à celle d'une croyance religieuse qui, elle, dispose du salut pour récompenser une manière de vivre particulière ${ }^{102}$. 


\section{NOTES}

1. P. Besnard, Protestantisme et capitalisme, Paris, 1970, p. 57.

2. Max Weber, L'éthique protestante et l'esprit du capitalisme, Paris, 1964, p. 81.

3. W. Sombart, Les juifs et la vie économique, Paris, 1923, p. 320.

4. Ibid., p. 251.

5. Marianne Weber, Max Weber, ein Lebensbild, Tübingen, 1926, p. 289.

6. H. Burgelin, La société allemande, Paris, 1969, p. 118.

7. W. Dilthey, Introduction à l'étude des sciences humaines, Paris, 1942, p. 29.

8. W. Dilthey, Le monde de l'esprit, t. 1, Paris, 1947, pp. 271-272.

9. Max Weber, Essais sur la théorie de la science, Paris, 1965, p. 200.

10. Max Weber, Essais sur la théorie de la science, Paris, 1965, p. 117 sq.

11. J. Freund, «La neutralité axiologique » in Cahiers de l'I.S.M.E.A., Section M, $n^{\circ} 29,1977$, pp. 411-483.

12. J. Freund, « La neutralité axiologique... », op. cit., p. 459.

13. Ibid., p. 460.

14. Ibid.

15. Max Weber, Essais sur la théorie... op. cit., p. 418.

16. W. Sombart, Les juifs... op. cit. p. 13.

17. Ibid., p. 14

18. Max Weber, Essais sur la théorie de la science... op. cit., p. 183.

19. J. Freund, Les théories..., p. 108 sq.

20. G. Simmel, Philosophie des Geldes, Leipzig, 1900, p. 135.

21. J. Freund, La neutralité axiologique..., op. cit., pp. 445-446.

22. Max Weber, Economie... op. cit., t. 1, p. 18.

23. W. Sombart, op. cit., pp. 245-246.

24. Ibid., p. 55.

25. Ibid., p. 56.

26. Ibid., p. 233.

27. J. Freund, « La ville selon Max Weber », Espaces et sociétés, 16, nov. 1975, p. 48.

28. K. Marx, - F. Engels, L'idéologie allemande, Paris, 1968, p. 51.

29. J. Freund, «La prise de conscience économique et le facteur religieux », in Colloque de Louvain, déc. 1972 , p. 39.

30. Verhandlungen des ersten deutschen Soziologentages, Tübingen, 1911, p. 101.

31. Max Weber, Essais sur la théorie de la science, Paris, 1965, p. 200.

32. J. Freund, Max Weber, Paris, 1969, p. 44.

33. Ibid.

34. J. Freund, Religion et économie... op. cit., p. 29.

35. Raymond Aron, Les étapes... op. cit., p. 540.

36. Max Weber, L'éthique... op. cit., pp. 248-249.

37. J. Freund, Sociologie de M. Weber... op. cit., p. 182.

38. Max Weber, "Morale économique des grandes religions", trad. de M. Rubel, in Archives de sociologie des religions, IX (1960), pp. 3-30.

39. Cf. Aufsätze zur Religionssoziologie, op. cit., t. 1, p. 252.

40. Max Weber, L'éthique... op. cit., 1964, p. 107. Cit. par P. Besnard, op. cit. p. 18.

41. Max Weber, L'éthique... op. cit., p. 248. 
42. Ibid., p. 233.

43. P. Besnard, op. cit., p. 21.

44. W. Sombart, Der moderne Kapitalismus, t. 1, Leipzig, 1902, p. XXIX.

45. W. Sombart, Le bourgeois... op. cit., p. 327 sq.

46. Ibid., p. 328.

47. Ibid., p. 329.

48. Ibid., p. 330.

49. W. Sombart, Le bourgeois, op. cit., p. 334.

50. Ibid., pp. 330-331.

51. Ibid., p. 331.

52. L. Febvre, Pour une histoire à part entière, Paris, 1962, p. 327.

53. Max Weber, L'éthique protestante et l'esprit du capitalisme, Paris, 1964, p. 48.

54. Jean Baechler. Les origines du capitalisme, Paris, 1971, p. 116.

55. J. Freund, Religion et économie, Louvain, 1972, p. 20.

56. Max Weber, Wirtschaftsgeschichte. Abriß der universalen Sozial- und Wirtschaftsgeschichte, Berlin, 1958, p. 311.

57. Max Weber, L'éthique... op. cit., p. 72.

58. Philippe Besnard, Protestantisme et capitalisme, Paris, 1970, p. 79.

59. R. Aron, La sociologie allemande, op. cit., p. 118.

60. Max Weber, L'éthique... op. cit, 1964, p. 75.

61. R. Aron, Les étapes, op. cit., pp. 534-535.

62. Ibid., p. 534.

63. 2e édit., Berlin, 1909, p. 107.

64. Ibid., p. 113.

65. P. Besnard, op. cit., p. 60.

66. R. Aron, La sociologie allemande contemporaine, 3e édit., Paris, 1966, p. 114.

67. W. Sombart, Le bourgeois, Paris, 1926, p. 313.

68. Ibid., p. 329.

69. W. Sombart, Les juifs..., op. cit., p. 203.

70. Ibid., p. 211.

71. Ibid., p. 212.

72. W. Sombart, L'apogée du capitalisme, Paris, 1932, p. 44.

73. P. Besnard, Protestantisme et capitalisme, Paris, 1970, p. 60.

74. W. Sombart, Les juifs... op. cit., p. 218.

75. Ibid. p. 220.

76. Ibid., p. 214.

77. Ibid. p. 215.

78. W. Sombart, Les juifs... op. cit., pp. 221-222.

79. J. Le Goff, Marchands et banquiers du Moyen Age, 5e édit., Paris, 1972, p. 39.

80. Ibid., p. 40.

81. Ibid., p. 41.

82. W. Sombart, Les juifs..., op. cit., p. 454-455

83. Ibid., p. 454.

84. Ibid., p. 455.

85. W. Sombart. Le bourgeois... op. cit., p. 402.

86. Ibid., p. 407.

87. Ibid., p. 332-333.

88. Ibid., p. 318.

89. W. Sombart, Les juifs... op. cit., p. 203.

90. W. Sombart. Le bourgeois... op. cit., p. 289. 
91. Ibid., p. 294.

92. Ibid., p. 303 sq.

93. Ibid., p. 309.

94. Ibid., p. 313.

95. Ibid., pp. 314-315.

96. Ibid., p. 315.

97. H. M. Robertson, Aspects of the Rise of Economic Individualism, London, 1933.

98. P. Besnard, Protestantisme et capitalisme, Paris, 1970, p. 33.

99. Max Weber, L'éthique... op. cit., 1964, p. 17.

100. P. Besnard, op. cit., p. 37.

101. Max Weber, L'éthique... op. cit., 1964, p. 58.

102. Ibid. 